\title{
¿EXISTE EL VOTO PROGRAMÁTICO EN ELECCIONES CON UN SISTEMA DE PARTIDOS POLÍTICOS DÉBIL? UN ANÁLISIS DE LAS ELECCIONES PRESIDENCIALES PERUANAS DE 2016
}

\author{
Is There a Programmatic Vote in Elections in Weak Political Party \\ Systems? An Analysis of the 2016 Peruvian Presidential Election
}

\section{DAVID SULMONT}

Pontificia Universidad Católica del Perú

\begin{abstract}
RESUMEN
Este trabajo analiza la importancia que tienen las diferencias programáticas entre los partidos políticos para orientar el voto de los ciudadanos. Incluso en circunstancias donde existe un bajo nivel de institucionalización del sistema de partidos y una alta volatilidad electoral, los electores tienen la capacidad de diferenciar las posiciones que toman los políticos sobre temas controversiales y decidir su voto en función de sus propias preferencias. Se tomará como caso de estudio las elecciones presidenciales peruanas de 2016, analizando la relevancia de tres dimensiones y actitudes políticas en la decisión electoral: las orientaciones izquierda-derecha; las actitudes hacia la intervención del Estado en la economía ("estatismo económico"); las actitudes hacia el ejercicio discrecional del poder político en el gobierno ("mano dura").
\end{abstract}

Palabras clave: Perú, comportamiento electoral, voto programático, actitudes políticas

\begin{abstract}
This paper analyzes the importance of programmatic differences between political parties in guiding electoral choices among voters. Even in circumstances of low party system institutionalization and high electoral volatility, citizens can differentiate politicians' different programmatic stances on controversial issues and decide their vote according to their own preferences. Using the Peruvian presidential elections of 2016 as a case study, the study analyzed the relevance of three political dimensions and attitudes for electoral choice: leftwing orientations; attitudes toward government intervention in the economy ("economic statism"); and attitudes toward the discretional use of political power in government ("firm hand").
\end{abstract}

Keywords: Peru, electoral behavior, programmatic voting, political attitudes 


\section{INTRODUCCIÓN}

Idealmente, en una democracia los partidos políticos tratan de representar las preferencias de determinados segmentos del electorado respecto de los principales problemas sociales en sus idearios y programas políticos y electorales. Puede decirse que los electores votan "programáticamente" cuando orientan su voto evaluando la cercanía o lejanía de los programas electorales respecto de sus propias preferencias. Si a lo largo de elecciones sucesivas los partidos mantienen cierta consistencia en sus programas al mismo tiempo que se diferencian claramente de sus competidores, se van generando "vínculos programáticos" con una base electoral, lo que contribuye a la institucionalización de un sistema de partidos que ofrece pistas claras a los votantes (ideologías políticas y programas electorales) para que estos tomen una decisión que sea congruente con sus preferencias o intereses.

En sociedades con partidos políticos poco institucionalizados, altamente personalistas y con una alta volatilidad electoral, los vínculos programáticos serían difíciles de construir, por lo que el elector se orientaría su decisión a partir de las cualidades carismáticas de los políticos, promesas populistas o relaciones de tipo de clientelista. Sin embargo, la necesidad de diferenciarse entre sí ante el electorado puede forzar a los políticos a tomar posición respecto de los problemas que preocupan a los ciudadanos y sobre los cuales estos últimos pueden tener preferencias o intereses divergentes. Si esto es así, aun en contextos de baja institucionalización partidaria y alta volatilidad electoral, el comportamiento programático estaría presente en los votantes.

En este trabajo abordaremos estos temas analizando el caso de las elecciones presidenciales de Perú que tuvieron lugar entre abril y junio de 2016, en las que resultó ganador Pedro Pablo Kuczynski, del partido "Peruanos Por el Kambio", derrotando en la segunda vuelta electoral a su contendiente Keiko Fujimori, de "Fuerza Popular", por un estrecho margen de 0,24\% de votos válidos. El Perú es un caso que tiene un sistema de partidos bastante débil y poco institucionalizado, así como altos índices de volatilidad electoral, por lo que puede ser útil evaluar hasta qué punto se puede presentar un voto programático en un contexto teóricamente hostil. Por otro lado, al ser un proceso con dos vueltas electorales, además nos interesa analizar si los temas que diferenciaron la oferta electoral en la primera vuelta también lo hicieron en la segunda, especialmente en el caso de aquellos votantes cuyos candidatos no pasaron la valla electoral (más del 50\% de votos válidos).

Para ello se examinará cómo se posicionaron ideológica y programáticamente los principales candidatos presidenciales ante los ojos del electorado y hasta qué punto ello se relaciona con las preferencias de los votantes y su decisión electoral. Esto será evaluado en tres dimensiones que, consideramos, resultan relevantes en el contexto peruano: el papel del Estado en la regulación de la economía (estatismo económico); la discrecionalidad del ejercicio del poder 
y el respeto de normas constitucionales, libertades civiles y políticas; y la diferenciación izquierda-derecha.

Otra variable considerada en el análisis serán las actitudes políticas hacia el fujimorismo, especialmente en el marco de la segunda vuelta electoral. El fujimorismo es el movimiento político que ha logrado desarrollar una identificación política relativamente importante en el electorado peruano, al mismo tiempo que ha generado una fuerte oposición de varios sectores de la sociedad, algo que autores como Cyr y Meléndez (2016) han llamado una "antiidentidad política", y que habría sido central en la derrota electoral de Keiko Fujimori en las dos últimas elecciones presidenciales de 2011 y 2016.

El trabajo utilizará la información de una encuesta representativa a nivel nacional aplicada en mayo de 2016, dos semanas después de la primera vuelta de las elecciones presidenciales por el Instituto de Opinión Pública de la Pontificia Universidad Católica del Perú (IOP-PUCP). ${ }^{1}$ La encuesta es parte del proyecto Comparative Study of Electoral Systems (CSES), que incluye al Perú en su muestra desde las elecciones de 2001. Además de los módulos de preguntas propios del proyecto CSES, en la encuesta del IOP-PUCP se incluyó un módulo especialmente diseñado para evaluar las dimensiones de estatismo económico y discrecionalidad. ${ }^{2}$

En la primera parte del trabajo se discutirá la relación entre los vínculos programáticos y el comportamiento electoral y la manera en que esta podría presentarse en una sociedad como la peruana. Seguidamente se describirá brevemente el contexto y las candidaturas presentadas en las elecciones peruanas de 2016. Luego se dará cuenta de los datos empleados en este trabajo y la forma en que se han operacionalizado las variables de análisis. En la penúltima sección se presentarán los resultados empíricos del análisis: cómo se diferencian los candidatos y los electores en las dimensiones propuestas y cuál es el efecto que ello puede tener en las preferencias políticas y el voto, tanto en la primera vuelta como la segunda vuelta electoral. Finalmente, en las conclusiones se presentará un resumen de los principales hallazgos, discutiendo cuál es la utilidad y las limitaciones de un enfoque programático para el análisis del comportamiento electoral en una sociedad como la peruana.

1 La encuesta forma parte del proyecto de investigación 2016 del Grupo de Investigación sobre Comportamiento del Elector y Procesos Electorales de la PUCP, financiado con fondos del Vicerrectorado de Investigación de la PUCP.

2 Sobre el proyecto CSES, véase: http://www.cses.org y su repositorio de datos. Se prevé que el conjunto de los datos, incluyendo las variables específicas de este trabajo, estén disponibles al público general en el transcurso del 2019 a través del repositorio del IOP-PUCP: http:/ /iop-data.pucp.edu.pe. 


\section{VÍNCULOS PROGRAMÁTICOS Y COMPORTAMIENTO ELECTORAL}

Según Kitschelt y Wilkinson (2007a: 1-2), tanto los enfoques históricos comparados de los estudios electorales basados en los trabajos de Lipset y Rokkan (1967b), así como aquellos que siguen las teorías de elección racional inspiradas en el trabajo de Downs (1957), ${ }^{3}$ comparten algunos supuestos sobre el funcionamiento de la política democrática que enfatizan la importancia de los vínculos programáticos para lograr un gobierno de políticos que sean responsables ante sus ciudadanos, específicamente: a) los ciudadanos tienen preferencias sobre un conjunto de temas relevantes en la sociedad; b) los políticos agregan posiciones frente a esos temas en promesas y programas electorales, las cuales pueden representarse en algunas dimensiones clave (como izquierda-derecha) para simplificar el trabajo de búsqueda de información del elector; c) los votantes comparan sus preferencias con la oferta de los políticos, ponderándolas por la viabilidad electoral de los candidatos; d) los partidos que ganan tratan de implementar su promesas; e) en elecciones consecutivas los votantes responsabilizan a los políticos y a la oposición por su desempeño.

De acuerdo con Baker y Greene (2015: 173), varios analistas sostienen que en las "nuevas democracias" como las de América Latina, los vínculos programáticos entre políticos y ciudadanos son bastante débiles e inexistentes, y lo que prima más bien son conexiones basadas en ofertas populistas, relaciones clientelistas ${ }^{4}$ o carismáticas. Los vínculos programáticos serían más bien un atributo de sistemas de partidos más estables y construidos sobre estructuras de clivajes que articulan sectores de la ciudadanía y sus conflictos sociales centrales, con instituciones representativas y la competencia electoral (Lipset y Rokkan 1967a; Dogan 1996; Bartolini 2005; Bornschier 2009), lo que permitiría una mayor predictibilidad respecto del comportamiento electoral. En varios países de América Latina estas conexiones han sido difíciles de construir debido a la heterogeneidad estructural y la escasa consolidación de instituciones democráticas hasta casi finales del siglo XX (Roberts 2002).

Sin embargo, contrariamente a las expectativas negativas sobre el voto programático en América Latina, Baker y Greene (2015) afirman que existen varios indicios de que en la región los electores efectivamente votan en función de algunas posiciones que diferencian tanto las preferencias de los ciudadanos como las propuestas programáticas de los políticos sobre temas controvertidos ("position issues"), además de que este tipo de voto puede distinguirse de evaluaciones respecto de las cualidades personales de los candidatos. En su trabajo, Baker y Greene exploran tres temas sobre los cuales los electores y políticos latinoamericanos pueden mostrar posiciones diferentes que tendrían relevancia electoral: a) el desarrollo económico y el rol del gobierno

Véase por ejemplo: Merrill y Grofman (1999); Adams et al. (2005).

Sobre los vínculos clientelistas, véase: Kitschelt (2000) y Kitschelt y Wilkinson (2007b). 
en la economía; b) los medios a través de los cuales el gobierno combate a la delincuencia; c) el grado en el cual el Estado regula temas relativos a la moral personal (sexualidad, familia, relaciones de género). Según los autores, cuando los candidatos debaten y se diferencian sobre estos temas, en ciertos países los electores reaccionan "programáticamente" al votar considerando sus propias preferencias.

\section{La dimensión izquierda-derecha}

La diferenciación entre izquierda y derecha ha sido uno de los mecanismos clásicos a través de los cuales se han representado y resumido vínculos programáticos entre electores y políticos en diferentes países. Esta se convierte en un mecanismo de heurística electoral o "atajo cognitivo" que "economiza" el trabajo de decisión del elector al agregar en un solo eje un conjunto de posiciones programáticas de los partidos. Sin embargo, varias investigaciones han encontrado que tanto la importancia como el contenido específico las posiciones izquierda-derecha varía mucho de país en país y de elección en elección (Inglehart y Klingemann 1976; Martin 2000; Badescu y Sum 2005; Norris 2006; Lachat 2008; Best y McDonald 2010; Corral y Zechmeister 2010; Dalton 2010; Sulmont 2015; Zechmeister 2010, 2015).

De acuerdo con Dalton (2010: 105), históricamente las diferencias entre izquierda y derecha han estado relacionadas con diversas posiciones respecto de conflictos económicos y de clase. Las posiciones de izquierda se asocian con una mayor participación y rol de regulador del Estado en la economía, así como con un énfasis en la reducción de las desigualdades sociales. Por su parte, las posiciones de derecha se orientan hacia políticas más favorables a la liberalización del mercado, el rol central de la inversión y las empresas privadas en la economía, y un Estado más reducido o limitado. Sin embargo, a lo largo del siglo XX, la distinción izquierda-derecha también ha integrado posiciones divergentes respecto de temas como la moral social, la religión, la ecología, la igualdad de género y el multiculturalismo (Dalton 2010: 106). En algunos países de América Latina que han experimentado dictaduras militares o regímenes autoritarios, las posiciones de izquierda se han asociado con la defensa de los derechos humanos, mientras que las de derecha con actitudes más represivas de la protesta social y más proclives al uso de la "mano dura" para mantener el "orden social" (Zechmeister 2015).

La variedad de significados de los ejes de izquierda-derecha indica que esta dimensión no corresponde necesariamente a una profunda y consistente ideologización de la ciudadanía, sino que más bien depende la manera en cómo los políticos presentan la oferta programática ante el electorado y se diferencian entre sí, "activando" o "desactivando" este tipo de atajos cognitivos (Martin 2000: 18-21; Sniderman y Levendusky 2007: 349-341). El nivel de institucionalización, la complejidad y cantidad de partidos, así como el grado 
de polarización ideológica y programática de los partidos políticos tiene efectos importantes en estos procesos (Lachat 2008; Kroh 2009; Best y McDonald 2010; Dalton 2010). En el caso de América Latina, trabajos como los de Zechmeister (2015) y Sulmont (2015) muestran que en los países que tienen sistemas de partidos más estructurados y polarizados ideológicamente (como por ejemplo, Chile y Uruguay), los votantes tienen mayor claridad sobre las alternativas en conflicto y el significado de las posiciones de izquierda o derecha. Cuando no es así (como en muchos de los países de la región) la dimensión izquierda-derecha pierde significancia para el votante y su decisión se vuelve más impredecible. Por otro lado, en varios estudios se ha encontrado que la capacidad del elector de utilizar esta dimensión para evaluar la oferta política depende en gran medida de su nivel educativo y estatus social, así como de su interés y grado de sofisticación política (Lachat 2008; Dalton 2010; Sulmont 2015; Zechmeister 2015).

\section{Dimensiones programáticas relevantes en la sociedad peruana}

Deacuerdocon varios autores, el Perú resalta por ser un país con serias dificultades para consolidar un sistema de partidos más estable e institucionalizado. A fines del siglo XX, se consideraba que el Perú tenía un sistema de partidos con una "baja o nula" estructuración en base a vínculos programáticos (Kitschelt et al. 2010: 320), lo que se habría visto agravado en los años siguientes al punto de considerarse que el Perú es un caso de una "democracia sin sistema de partidos" (Levitsky y Cameron 2013; Tanaka 2005). Lo que primaría en la actualidad son partidos altamente personalistas o formados a partir de la agregación oportunista de personas que quieren tener una carrera política pero que no tienen muchos incentivos para permanecer leales a una organización, dada la alta volatilidad electoral predominante en el sistema (Zavaleta 2014).

En estas condiciones no se esperaría que el votante peruano tome en cuenta evaluaciones programáticas para decidir su voto. Sin embargo, autores como Carlos Meléndez sostienen que, a pesar de seguir funcionando sobre la base de estructuras partidarias personalistas, algunos actores políticos que logran cierta permanencia en la competencia electoral peruana han desarrollado un posicionamiento ideológico identificable por los ciudadanos sobre la base de una consistencia discursiva respecto de determinados temas o problemas sociales (Meléndez 2012: 24-26).

En un ensayo publicado luego de las elecciones presidenciales de 2006, Alberto Vergara (2007) sostiene que las alternativas programáticas que han marcado la oferta política peruana desde finales del régimen fujimorista pueden ubicarse en dos ejes superpuestos: uno institucional y otro económico. En el eje institucional, los actores políticos se diferencian en función de su respeto relativo por las reglas formales del juego democrático (las libertades individuales y el balance de poderes). Los extremos varían entre un mayor respeto a las reglas y la ley, a 
una mayor discrecionalidad respecto de los principios constitutivos del Estado de Derecho y el ejercicio del poder.

Por su lado, en el eje económico se distingue un extremo favorable a la promoción de la libre competencia en el mercado de las empresas privadas como motor del desarrollo económico; otro extremo representaría posiciones favorables a la intervención del Estado en la economía para asegurar un crecimiento socialmente más inclusivo, que redistribuya la riqueza y asegure un mayor control de los recursos naturales por parte de la Nación. El primero de los extremos es llamado "librecambismo", mientras que el extremo opuesto recibe la etiqueta de "intervención estatal".

De acuerdo con Vergara, la historia política y social del Perú republicano muestra la escasa consolidación de instituciones del Estado de Derecho y de orientaciones democrático-liberales en el eje institucional. Más bien, lo que predominaría es un mayor peso de la dimensión discrecional, sobre la cual se apoyarían propuestas programáticas de corte populista-autoritario, ya sea de tipo redistributivo estatista o que apuestan por la liberalización económica, pero sin demasiado énfasis en la consolidación de un Estado de derecho democrático.

El peso gravitante y creciente del fujimorismo en la política electoral peruana, así como las polémicas en torno a los legados autoritarios del gobierno de Alberto Fujimori (1990-2000), constituiría otro de los elementos que estructuraría el debate político en el Perú contemporáneo. Carlos Meléndez (2012, 2013) considera que la supervivencia y creciente arraigo electoral que el fujimorismo ha logrado en las elecciones peruanas desde el 2006, han venido acompañadas de una relativa consistencia discursiva e identificación programática de derecha que justifica medidas de mano dura o de corte autoritario frente a problemas percibidos como amenazas por la sociedad (inestabilidad económica, terrorismo, delincuencia). En su evaluación de las elecciones de 2006 (en donde Keiko Fujimori se presentó por primera vez como candidata al parlamento), Vergara ubica al fujimorismo en el extremo "discrecional" del eje institucional y en el lado "librecambista" del eje económico.

Cyr y Meléndez han sostenido que la consolidación de la identidad política fujimorista estaría sirviendo en la política peruana contemporánea de eje de diferenciación de la oferta política, puesto que a la par del fujimorismo, se habría desarrollado también una "anti-identidad" política, el "antifujimorismo" (Cyr y Meléndez 2016: 214) que tiene características sociodemográficas, ideológicas y actitudinales claramente opuestas al fujimorismo, y que habría sido clave en la derrota de la candidata Keiko Fujimori en la segunda vuelta de las dos elecciones presidenciales en las que se presentó: ante Ollanta Humala en 2011 y ante PPK en 2016. 


\section{EL CONTEXTO Y LA OFERTA DE LAS ELECCIONES PERUANAS DE 2016}

Las elecciones generales de 2016 se desarrollaron en el marco de una fuerte erosión del apoyo al gobierno de Ollanta Humala en la opinión pública. Un mes antes de las elecciones, en marzo de 2016, la aprobación presidencial se situaba alrededor del 14\% (Instituto de Opinión Pública - PUCP 2016). Según la encuesta preelectoral del IOP-PUCP de marzo de 2016, más del $40 \%$ de los peruanos seguía reclamando cambios radicales en el manejo de la economía ( $7 \%$ más que lo registrado en una encuesta similar en 2011), y tan solo 13\% estaba de acuerdo con mantener la orientación de la política económica del gobierno ( $9 \%$ menos que cinco años atrás) (Instituto de Opinión Pública - PUCP 2016).

A pesar de este contexto, la oferta política que se presentó en las elecciones presidenciales de 2016 guardaba varias semejanzas con la de 2011. Entre los tres candidatos con mayores opciones según las encuestas de opinión, dos habían ya participado en las elecciones de 2011: Keiko Fujimori y PPK, obteniendo respectivamente la segunda y tercera mayor votación en la primera vuelta en esa oportunidad. Tanto en 2011 como en 2016, ambos representaban diversas posiciones programáticas ubicadas a la derecha del espectro político, con algunas diferencias importantes. Desde mediados de 2015, Fujimori registraba intenciones de voto superiores al 33\% según las principales encuestadoras del país (Ipsos Opinión y Mercado 2016b). Su campaña tuvo como un eje importante el tema de la inseguridad ciudadana, frente al cual se presentaba como la alternativa capaz de enfrentarlo con mayor firmeza y "mano dura". 5

Por su lado, PPK es un antiguo miembro del "establishment" político, ha sido ministro de Energía y Minas en el gobierno de Fernando Belaúnde (1980-1985), ministro de Economía y presidente del Consejo de Ministros durante el gobierno de Alejandro Toledo (2001-2006). Como economista, PPK ha apoyado varias de las tesis del llamado "Consenso de Washington" que buscaron liberalizar la economía de las sociedades latinoamericanas desde finales de la década de 1980 (Balassa et al. 1986; Williamson y Kuczynski 2003), por lo que se lo podría calificar como un liberal en materia económica, pero que durante su campaña (especialmente en la segunda vuelta), buscó diferenciarse de Fujimori en cuanto al respeto de las instituciones democráticas.

La tercera candidata importante en 2016 fue Verónika Mendoza, representando al Frente Amplio, una nueva agrupación que reunió a diversas organizaciones políticas de izquierda socialista y ecologista. A inicios de la campaña, Mendoza era una candidata poco conocida por el electorado y apenas lograba un $2 \%$ de las preferencias electorales en enero de 2016 (Ipsos Opinión y Mercado 2016a). En el último mes de la campaña la candidatura de Mendoza adquiere mayor 
visibilidad y empieza competir con Kuczynski por el segundo lugar de las preferencias electorales (Ipsos Opinión y Mercado 2016b).

Como se aprecia en la Tabla 1, la votación obtenida por Fujimori en la primera vuelta presidencial casi duplica la de Kuczynski, y esta última tiene un margen de ventaja muy estrecho respecto de la de Mendoza. En las últimas semanas de la elección, la diferencia registrada por los sondeos de opinión entre el segundo y tercer lugar estaba dentro de los márgenes de error muestrales (GfK 2016; Ipsos Opinión y Mercado 2016b) y hasta el mismo día de la elección existía la incertidumbre de quién acompañaría a Fujimori en la segunda vuelta presidencial.

Entre los demás candidatos "menores", Alfredo Barnechea de Acción Popular y Alan García de la Alianza Popular (APRA-PPC) representaban diversas posiciones de centro-derecha. Como se sabe García fue presidente de la República durante el periodo 2006-2011. Entre el resto de candidatos cabe destacar la figura de Gregorio Santos, quien se presentó con una plataforma de izquierda radical, pero que hizo campaña desde la cárcel, puesto que se encontraba en prisión preventiva acusado por hechos de corrupción ocurridos durante su gestión como gobernador regional de Cajamarca. También participó como candidato con poco éxito (1,3\% de los votos válidos) el expresidente (2001-2006) Alejandro Toledo, cuya agrupación Perú Posible perdió su inscripción al no pasar la valla electoral.

Tabla 1. Resultados de las Elecciones Presidenciales Peruanas 2016 y de la Encuesta Nacional Post Electoral PUCP

\begin{tabular}{|c|c|c|c|c|}
\hline & \multicolumn{2}{|c|}{$\begin{array}{l}\text { Resultados oficiales } \\
\text { ONPE (\% total) }\end{array}$} & \multicolumn{2}{|c|}{$\begin{array}{c}\text { Encuesta IOP-PUCP } \\
\text { del } 5 \text { al } 17 \text { mayo } \\
\text { (\% entrevistados válidos)* }\end{array}$} \\
\hline & 1ra vuelta & 2 da vuelta & \multirow{2}{*}{$\begin{array}{l}\text { Voto reportado } \\
\text { 1ra vuelta }\end{array}$} & \multirow{2}{*}{$\begin{array}{c}\text { Intención } \\
\text { de voto } 2 \text { da } \\
\text { vuelta }\end{array}$} \\
\hline Candidato & 10 de Abril & 5 de Junio & & \\
\hline Fujimori & 32,6 & 46,6 & 37,2 & 42,2 \\
\hline PPK & 17,2 & 46,9 & 19,6 & 36,9 \\
\hline Mendoza & 15,4 & & 17,5 & \\
\hline Barnechea & 5,7 & & 6,5 & \\
\hline García & 4,8 & & 5,4 & \\
\hline Santos & 3,3 & & 3,7 & \\
\hline Otros & 2,9 & & 3,3 & \\
\hline Blanco / viciado & 18,1 & 6,5 & 6,8 & 10,8 \\
\hline No sabe & & & & 10,1 \\
\hline Total \% & 100,0 & 100,0 & 100,0 & 100,0 \\
\hline $\mathrm{N}$ & 18.734 .130 & 18.342 .896 & 1.448 & 1.480 \\
\hline
\end{tabular}

Fuentes: Oficina Nacional de Procesos Electorales - ONPE (www.onpe.gob.pe); IOP-PUCP. Elaboración propia. ${ }^{*}$ \% ponderados según diseño muestral. 
Para ganar las elecciones en la segunda vuelta, PPK necesitaba atraer el voto de un electorado bastante heterogéneo, no sólo los electores de Barnechea y de García, sino sobre todo los que votaron por Mendoza, quienes se ubicaban en el lado opuesto del espectro político izquierda-derecha. En las siguientes secciones trataremos de evaluar hasta qué medida las actitudes políticas de los electores en las diferentes dimensiones que nos interesa analizar (izquierdaderecha, estatismo, discrecionalidad y fujimorismo / antifujimorismo) pueden haber jugado un rol en el resultado final.

\section{DATOS, NIVELES Y VARIABLES DE ANÁLISIS}

Como se indicó en la introducción, en este trabajo se utilizarán los datos producidos por la encuesta nacional realizada por el IOP-PUCP entre el 5 y el 17 de mayo de 2016, entre las dos rondas de las elecciones presidenciales. La muestra de 1574 personas es representativa de la población electoral residente en el país. El diseño muestral fue probabilístico, estratificado y polietápico; el margen de error estimado para la muestra total es de $\pm 2,5 \%$, con un nivel de confianza del 95\%. Las variables escogidas para el análisis operacionalizan las diferentes dimensiones de interés para este estudio: preferencias electorales, identificaciones con los candidatos, posiciones ideológicas izquierda-derecha, actitudes hacia el manejo económico y actitudes hacia el ejercicio discrecional del poder político.

Las principales variables dependientes para este estudio son las siguientes:

- Nivel de preferencia por los candidatos: mide en una escala de 0 a 100 el nivel de preferencia que tiene el elector por los principales candidatos presidenciales. $^{6}$

- Voto reportado en primera vuelta de las elecciones presidenciales.

- Intención de voto para la segunda vuelta electoral.

El análisis diferenciará dos niveles. En primer lugar, se evaluará la percepción que tiene la ciudadanía de los principales candidatos presidenciales respecto de tres dimensiones:

- El posicionamiento de los candidatos en el eje izquierda-derecha: para ello se utilizará una variable donde los entrevistados ubican cada candidato en una escala izquierda-derecha que varía entre 0 y 10 , donde $0=$ "extrema izquierda y 10 = extrema derecha".

La pregunta original en la encuesta le pide al entrevistado evaluar de 0 a 10 qué tanto le gusta cada uno de los candidatos presidenciales $(0=$ Nada; $10=$ Mucho). Para efectos del análisis, la variable original ha sido multiplicada por 10 para tener una escala porcentual. 
- La ubicación de los principales candidatos en un eje Mercado-Estado. Para ello en la encuesta se aplicó una pregunta donde se le pedía a los entrevistados que ubicaran a cada candidato en una escala donde: 0 = "a favor de una economía privada de mercado" y 10 = "a favor de una economía regulada por el Estado".

- El posicionamiento de los candidatos en un eje Respeto a la leyDiscrecionalidad. En este caso a los entrevistados se les preguntó: "Si [el candidato X] fuese presidente ¿cuál cree que sería su actuación para enfrentar una crisis?"; por cada candidato, el entrevistado debía responder considerando una escala donde $0=$ "Siempre respetará las leyes y la constitución" y 10 = "No siempre respetará las leyes y la constitución". 7

Para el análisis, los valores de todas estas escalas han sido multiplicados por 10 para que puedan ser interpretadas en una escala porcentual.

El segundo nivel de análisis se centrará en las actitudes de los electores y sus preferencias electorales; considerará, además de las variables previamente identificadas, el posicionamiento de los electores en:

- La escala izquierda-derecha.

- Una escala de actitudes estatistas respecto del manejo de la economía, donde $0=$ "nada estatista" y $100=$ "muy estatista".

- Una escala de actitudes hacia el ejercicio discrecional del poder, donde $0=$ "nada discrecional" y $100=$ "muy discrecional".

Las escalas de "Estatismo" y "Discrecionalidad" son escalas de actitudes de tipo Lickert que fueron especialmente diseñadas para esta investigación. Para ello inicialmente se probaron 30 ítems para cada dimensión ${ }^{8}$ en una muestra de 400 jueces, ${ }^{9}$ seleccionados mediante un muestreo probabilístico en distritos de Lima metropolitana representativos de dos estratos socioeconómicos: medio y medio-bajo. Luego del análisis se seleccionaron 8 ítems por escala entre aquellos que tenían mayor relación con la dimensión que se quería medir. ${ }^{10}$

Las versiones definitivas de las escalas fueron aplicadas en preguntas donde se les pedía a los entrevistados que indicaran su grado de acuerdo o en desacuerdo con cada ítem, usando 5 alternativas de respuesta ("Muy en desacuerdo", "De

7 En la encuesta, las preguntas originales consideran como 10 el extremo de "Mercado" y 0 el de "Estado"; y 10 para "Respeto a la ley" y 0 para "No respeto" (Discrecionalidad). Para efectos del análisis se ha invertido los valores, buscado una equivalencia con las escalas de estatismo y discrecionalidad elaboraras para analizar las actitudes del electorado.

8 En el caso de la escala de discrecionalidad, algunos ítems estuvieron inspirados en preguntas del Barómetro de las Américas relativas a actitudes iliberales (Carrión, Zárate y Zechmeister 2015)

9 La encuesta para validar las escalas de estatismo económico y discrecionalidad fue aplicada en marzo de 2016.

10 Para la construcción de las escalas y la selección de ítems se siguieron los procedimientos descritos por Padua y Ahman (1979), complementados por un análisis de componentes principales. 
acuerdo", "Ni de acuerdo ni en desacuerdo", "De acuerdo", "Muy de acuerdo"), que fueron codificadas del 0 al 4 . Para la versión final de cada escala se sumaron los valores de las respuestas a cada ítem, sumatoria que fue luego convertida en una escala de 0 a 100.

La validez y confiabilidad de las escalas fueron comprobadas mediante análisis de componentes principales y Alpha de Cronbach. En las tablas 2 y 3 puede apreciarse los ítems correspondientes a cada escala, así como la media de cada ítem (de 0 a 4) en la muestra de la encuesta nacional, se incluye además el peso (r de Pearson) del ítem en el primer componente principal (CP) y el Alpha de Cronbach. Como se observa, el primer componente principal representa más del $43 \%$ de la varianza compartida por los ítems en cada una de las escalas y los coeficientes $r$ de Pearson muestran correlaciones importantes con el primer componente principal extraído. Por otro lado, el Alpha de Cronbach en ambos casos es igual o superior a 0,8 , lo que indica un elevado nivel de confiabilidad de los ítems seleccionados.

Tabla 2. Ítems de la Escala de Estatismo Económico

\begin{tabular}{|c|c|c|}
\hline \multicolumn{3}{|l|}{ Análisis de Componentes Principales y Alpha de Cronbach } \\
\hline \multicolumn{3}{|c|}{ (Valores respuestas: Muy en desacuerdo $=0 ;$ Muy de acuerdo $=4$ ) } \\
\hline Ítem & $\begin{array}{l}\text { Media del } \\
\text { Ítem }\end{array}$ & $\begin{array}{l}\text { Peso en el } \\
\text { Primer CP }\end{array}$ \\
\hline $\begin{array}{l}\text { Las ganancias de las grandes empresas privadas debe- } \\
\text { rían ser mucho más controladas por el Estado. }\end{array}$ & 3,20 & 0,64 \\
\hline $\begin{array}{l}\text { La libertad de empresa sólo beneficia a los más podero- } \\
\text { sos. }\end{array}$ & 3,00 & 0,51 \\
\hline $\begin{array}{l}\text { El Estado debería intervenir más en la economía para } \\
\text { que la riqueza se reparta con mayor justicia. }\end{array}$ & 3,20 & 0,72 \\
\hline $\begin{array}{l}\text { Algunas grandes empresas privadas deberían estatizarse } \\
\text { para que sus recursos beneficien más a la mayoría de los } \\
\text { peruanos. }\end{array}$ & 2,90 & 0,66 \\
\hline $\begin{array}{l}\text { El Estado debería controlar los precios de los alimentos } \\
\text { para que no afecten la economía del pueblo. }\end{array}$ & 3,20 & 0,70 \\
\hline $\begin{array}{l}\text { Una mayor intervención del Estado en la economía es } \\
\text { la mejor forma en que el Perú puede desarrollarse. }\end{array}$ & 3,10 & 0,71 \\
\hline $\begin{array}{l}\text { Debería limitarse la propiedad de las tierras para que } \\
\text { puedan distribuirse de forma más justa entre los campe- } \\
\text { sinos. }\end{array}$ & 2,70 & 0,59 \\
\hline $\begin{array}{l}\text { Si el Estado controlase más empresas de servicios públi- } \\
\text { cos como telefonía o electricidad, los costos para el pue- } \\
\text { blo serían más justos. }\end{array}$ & 3,20 & 0,68 \\
\hline Proporción de la varianza común & & 0,43 \\
\hline Alpha de Cronbach & & 0,80 \\
\hline
\end{tabular}


Tabla 3. Ítems de la Escala de Discrecionalidad en el Ejercicio del Poder Político

\begin{tabular}{|c|c|c|}
\hline \multicolumn{3}{|l|}{ Análisis de Componentes Principales y Alpha de Cronbach } \\
\hline \multicolumn{3}{|c|}{$($ Valores respuestas: Muy en desacuerdo $=0 ;$ Muy de acuerdo $=4$ ) } \\
\hline Ítem & $\begin{array}{l}\text { Media del } \\
\text { Îtem }\end{array}$ & $\begin{array}{l}\text { Peso en el } \\
\text { Primer CP }\end{array}$ \\
\hline $\begin{array}{l}\text { La policía debería poder allanar una casa sin permiso } \\
\text { judicial para luchar contra la delincuencia. }\end{array}$ & 2,01 & 0,72 \\
\hline $\begin{array}{l}\text { El Estado debería poder revisar las comunicaciones per- } \\
\text { sonales sin permiso judicial para luchar contra la inse- } \\
\text { guridad ciudadana. }\end{array}$ & 2,12 & 0,76 \\
\hline $\begin{array}{l}\text { La policía debería poder detener a una persona el tiem- } \\
\text { po que considere necesario como medida para luchar } \\
\text { contra la delincuencia. }\end{array}$ & 2,49 & 0,70 \\
\hline $\begin{array}{l}\text { El Estado debería poder revisar las cuentas bancarias sin } \\
\text { permiso de un juez para luchar contra la delincuencia. }\end{array}$ & 2,22 & 0,71 \\
\hline $\begin{array}{l}\text { Cumplir con todas las leyes no es tan importante si un } \\
\text { político logra buenos resultados para el país }\end{array}$ & 1,90 & 0,59 \\
\hline $\begin{array}{l}\text { Para hacer cumplir la ley, las autoridades algunas veces } \\
\text { tienen que romper las reglas para llevar a los criminales } \\
\text { a la justicia. }\end{array}$ & 2,34 & 0,69 \\
\hline $\begin{array}{l}\text { Cuando el país enfrenta momentos muy difíciles debido } \\
\text { a la corrupción, es justificable que el presidente cierre el } \\
\text { Congreso y gobierne sin Congreso. }\end{array}$ & 1,77 & 0,53 \\
\hline $\begin{array}{l}\text { Las personas pueden hacer justicia por su propia cuenta } \\
\text { cuando el Estado no castiga a los criminales. }\end{array}$ & 2,10 & 0,54 \\
\hline Proporción de la varianza común & & 0,44 \\
\hline Alpha de Cronbach & & 0.81 \\
\hline
\end{tabular}

Adicionalmente, para evaluar el efecto del antifujimorismo en la intención de voto en la segunda vuelta electoral, se generó una variable dicotómica a partir de la variable "nivel de preferencia por Keiko Fujimori", donde los niveles de 40 hasta el 0 (cero), es decir el tercio inferior de valores de la escala, han sido recodificados como 1 (antifujimorismo) y el resto de valores como 0 (cero). 


\section{LAS POSICIONES PROGRAMÁTICAS DE LOS CANDIDATOS Y ELECTORES PERUANOS EN 2016}

\section{Los candidatos presidenciales y la percepción de sus posiciones políticas}

En el Gráfico 1 puede apreciarse cómo los encuestados han ubicado a los principales candidatos presidenciales en las tres dimensiones del primer nivel de análisis propuesto. En el caso de la dimensión izquierda-derecha, Fujimori y PPK son ubicados claramente por al lado derecho del espectro político, sin mayor diferenciación entre ellos, mientras que Mendoza aparece al lado izquierdo, ubicación que comparte con Santos. Los candidatos Barnechea y García son los que se ubican más al centro.

En la medida que la posición en esta escala forma parte de la batería de preguntas del módulo del proyecto CSES que se replica en cada elección, podemos comparar los resultados de la encuesta de 2016 con las de $2011 .{ }^{11} \mathrm{En}$ el Gráfico 2 se presenta la posición media en el eje izquierda-derecha de los tres principales candidatos que compitieron en ambas elecciones, así como el autoposicionamiento de los propios encuestados. Como puede apreciarse, en ambos casos Fujimori y PPK aparecen en el lado derecho del espectro político, aunque respecto de 2011, en 2016 estos dos candidatos se han desplazado más hacia la derecha, al igual que el elector promedio. Por su parte, Mendoza se encuentra del lado que previamente ocupó Humala, aunque algo más a la izquierda.

11 Al igual que en 2016, la encuesta del proyecto CSES en el 2011 se aplicó entre las dos vueltas electorales. La ejecución de ella también estuvo a cargo del IOP-PUCP. Los datos de 2011 pueden descargarse del repositorio del CSES en http:/ / www.cses.org. 
Gráfico 1. Posición de los candidatos en las escalas Izquierda - Derecha, Mercado - Estado y Respecto a la Ley (media e intervalo de confianza al 95\%)
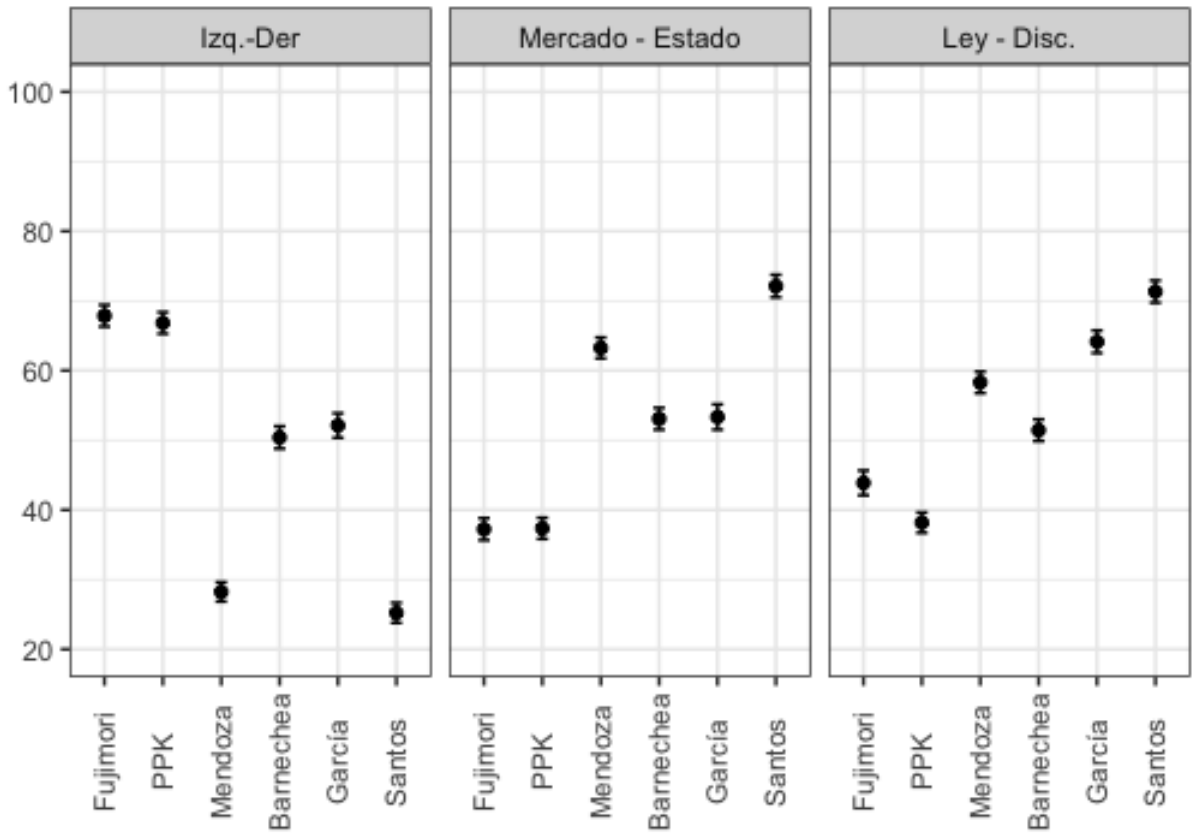

En cuanto a la dimensión mercado-Estado, en el Gráfico 1 se observa que tanto Fujimori como PPK son ubicados en el lado que podríamos denominar "librecambista" (usando la terminología de Vergara), sin que se aprecien diferencias estadísticamente significativas entre ellos; Mendoza y sobre todo Santos en el lado "estatista"; y Barnechea y García en posiciones intermedias, con ligera tendencia al lado "estatista". 
Gráfico 2. Posición del elector y de los principales candidatos presidenciales 2011 y 2016 en la escala Izquierda - Derecha (media e intervalo de confianza al 95\%)
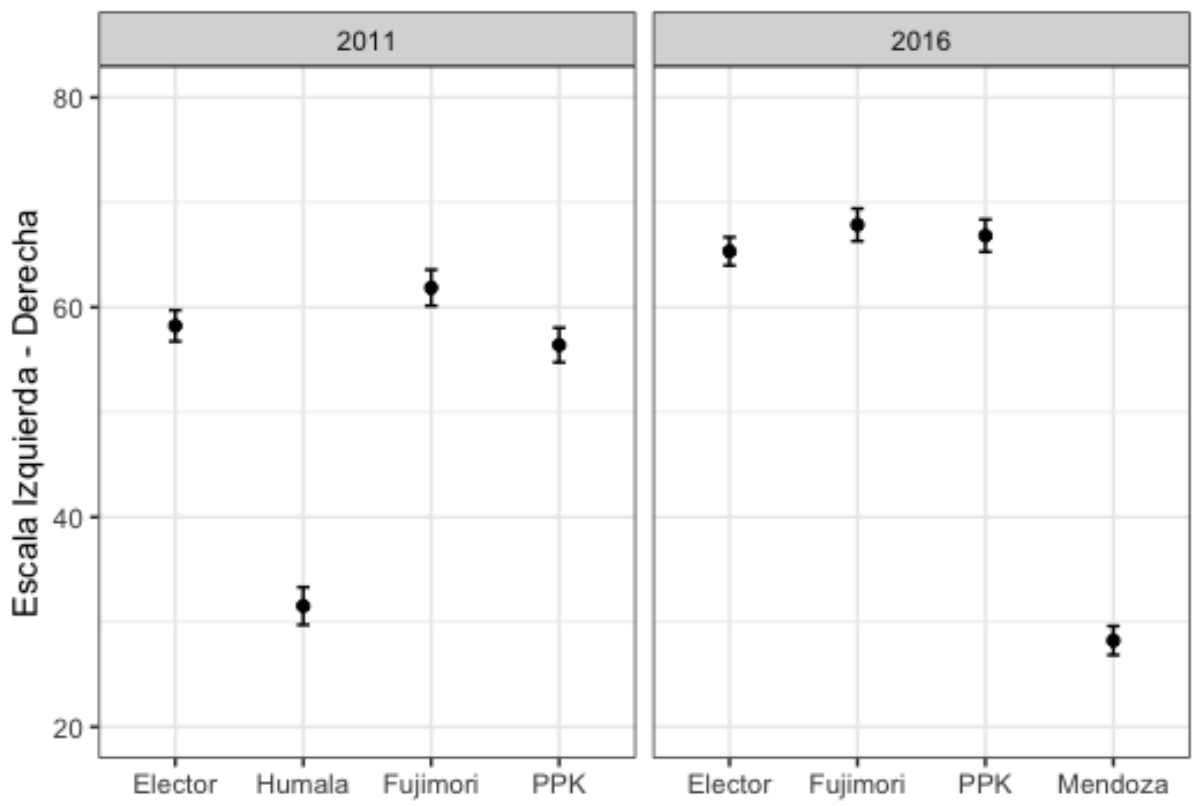

En la tercera dimensión, ley-discrecionalidad, todos los candidatos son ubicados en posiciones que mantienen diferencias estadísticamente significativas entre sí. PPK es el más cercano al extremo de "respecto a la ley", seguido por Fujimori. Mendoza se ubica en el lado opuesto de los dos primeros. García y Santos son percibidos como los candidatos que menos respetarían la ley y la constitución. Barnechea se ubica en una posición intermedia.

La manera en cómo los electores ubican a los candidatos en la dimensión izquierda- derecha está asociada con su posicionamiento en las otras dos dimensiones. Como puede verse en el Gráfico 1, los candidatos que se ubican hacia la derecha son percibidos como más favorables a la economía de libre mercado, mientras que los que están ubicados hacia la izquierda con posiciones estatistas. Por otro lado, los candidatos que son posicionados como de derecha, aparecen como más respetuosos de la ley, mientras que ocurre lo contrario con los ubicados a la izquierda. Esto se ve confirmado por las correlaciones entre estas variables que se muestran en la Tabla 4, donde nos concentramos en la forma en cómo han sido evaluados los tres principales candidatos. Se observa que también existe un nivel de asociación significativa entre ser percibido como proclive a una economía de mercado y ser respetuoso de la ley (o viceversa), especialmente en los casos de PPK y Mendoza. 
Tabla 4. Matriz de correlaciones r de Pearson entre las posiciones de los candidatos en las escalas de Izquierda - Derecha, Mercado - Estado y Respeto a la Ley, según candidato

\begin{tabular}{|c|c|c|c|c|c|c|}
\hline & \multicolumn{6}{|c|}{ Candidatos } \\
\hline & \multicolumn{2}{|c|}{ Fujimori } & \multicolumn{2}{|c|}{ PPK } & \multicolumn{2}{|c|}{ Mendoza } \\
\hline & $\begin{array}{l}\text { Mercado } \\
\text { - Estado }\end{array}$ & $\begin{array}{l}\text { Ley } \\
\text { - Disc. }\end{array}$ & $\begin{array}{l}\text { Mercado } \\
\text {-Estado }\end{array}$ & $\begin{array}{c}\text { Ley } \\
\text { - Disc. }\end{array}$ & $\begin{array}{l}\text { Mercado } \\
\text { - Estado }\end{array}$ & $\begin{array}{c}\text { Ley } \\
\text { - Disc. }\end{array}$ \\
\hline $\begin{array}{l}\text { Izquierda } \\
\text { - derecha }\end{array}$ & $-0,32^{* * *}$ & $-0,34^{* * *}$ & $-0,41^{* * *}$ & $-0,39^{* * *}$ & $-0,40^{* * *}$ & $-0,35^{* * *}$ \\
\hline $\begin{array}{l}\text { Mercado } \\
\text { - Estado }\end{array}$ & & $0,21^{* * *}$ & & $0,38^{* * *}$ & & $0,34^{* * *}$ \\
\hline
\end{tabular}

Notas: ${ }^{*} \mathrm{p}<0,05 ;{ }^{* *} \mathrm{p}<0,01 ;{ }^{* * *} \mathrm{p}<0,001$

En esta primera etapa del análisis podemos resaltar tres hallazgos. En primer lugar, los electores son capaces de diferenciar posiciones programáticas de los candidatos y que, por lo menos en cuanto a las dimensiones izquierda-derecha y mercado-Estado, su posicionamiento corresponde en general a las señales que los propios candidatos dan al respecto y les reconocen observadores "expertos" o los analistas políticos. ${ }^{12}$ Ello abona a favor de la hipótesis de que el electorado peruano maneja un mínimo de información relevante para diferenciar programáticamente y "correctamente" la oferta política que tiene ante sí.

En segundo lugar, respecto de la dimensión ley-discrecionalidad, la caracterización que se hace del fujimorismo por parte de los analistas políticos que hemos citado nos llevaría a esperar una evaluación por parte del electorado más inclinada hacia el lado discrecional. Sin embargo, como veremos en la siguiente sección, es posible que ello se deba a un problema de endogeneidad: al ser Fujimori la candidata con mayor votación (el doble que su rival más cercano), es esperable que los electores que se inclinaron por ella le atribuyan fuertes características positivas, y el "respeto a la ley", tal y como fue fraseado en la pregunta de la encuesta, puede considerarse como tal. Sin embargo, resulta relevante que pese a ser la candidata más popular, su ubicación en esta dimensión esté más hacia el lado discrecional que la de PPK, lo que pudo tener efectos significativos en la decisión electoral en segunda vuelta asociados a la movilización del "antifujimorismo" en ese tramo de la elección.

Finalmente, en tercer lugar, si bien el contenido de la dimensión izquierdaderecha puede variar de país en país y de elección en elección, en el caso de los candidatos peruanos encontramos asociaciones "clásicas" entre izquierdaderecha y posiciones respecto del rol del Estado y el libre mercado en la 
economía de la sociedad: derecha pro mercado e izquierda pro Estado. El otro significado de la "derecha" está asociado con el "respeto a la ley", en contraste con los candidatos ubicados a la izquierda que son vistos como más proclives a no ceñirse a ella.

Estos hallazgos apoyarían la hipótesis de que en las elecciones peruanas de 2016 se presentaron algunos de los requisitos necesarios para postular la existencia de un razonamiento o un voto programático por parte del elector.

\section{Los electores, sus actitudes políticas y preferencias políticas}

En este segundo nivel de análisis se evaluará el resultado de las escalas que permiten ubicar a los electores en las dimensiones de izquierda-derecha, estatismo y discrecionalidad y su relación con las preferencias electorales. En el siguiente gráfico de cajas, se presenta la distribución de los valores de las escalas correspondientes a estas variables. Se aprecia claramente cómo el electorado peruano de 2016 está inclinado hacia la derecha, sólo un $25 \%$ presenta posiciones el centroizquierda o izquierda. También se observa una marcada distribución asimétrica negativa de la escala de estatismo, lo que indica que la gran mayoría de entrevistados se inclinan hacia actitudes estatistas. La dimensión de discrecionalidad muestra una distribución más normal respecto de las otras variables, pero con una cierta tendencia hacia actitudes más favorables a la discrecionalidad.

Gráfico 3. Distribución de valores de las escalas de Izquierda - Derecha, Estatismo y Discrecionalidad

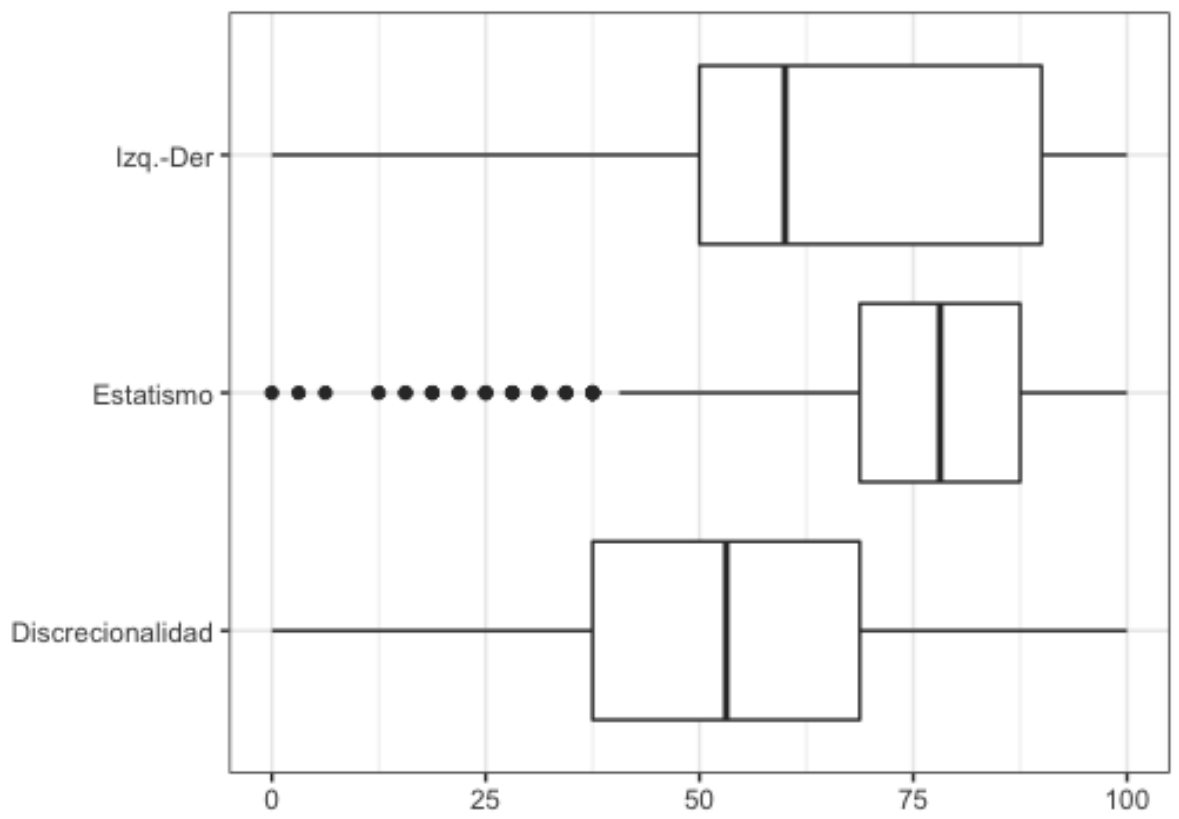


La siguiente tabla muestra los coeficientes de correlación entre los puntajes de las tres escalas. A diferencia de las dimensiones análogas correspondientes a ubicación de los candidatos, cuando se miden las actitudes de los electores, no aparecen relaciones muy importantes entre ellas. Es especialmente relevante la muy débil correlación entre la escala de estatismo y la de izquierda-derecha, lo que contrasta fuertemente con los resultados obtenidos en la sección precedente. Por otro lado, de forma parecida a las escalas que evalúan a los candidatos, actitudes estatistas de los electores en el ámbito económico, tienden a estar vinculadas con actitudes discrecionales en el ejercicio del poder, aunque el valor de coeficiente señala que se trata de una correlación algo débil y por lo tanto que ambas dimensiones pueden diferenciarse tanto teórica como empíricamente.

Tabla 5. Matriz de correlaciones r de Pearson entre las escalas de Izquierda Derecha, Estatismo y Discrecionalidad

\begin{tabular}{lcc}
\hline & Estatismo & Discrecionalidad \\
\hline Izquierda - Derecha & $-0,06 *$ & $-0,03$ \\
Estatismo & & $0,27 \quad * * *$ \\
\hline Notas: ${ }^{*} \mathrm{p}<0,05 ;{ }^{* *} \mathrm{p}<0,01 ;{ }^{* * *} \mathrm{p}<0,001$ & &
\end{tabular}

En el Gráfico 4 puede verse el puntaje de los electores en las tres dimensiones que estamos analizando, según el voto reportado en primera vuelta. Las diferencias más importantes se encuentran en la escala izquierda - derecha. Las posiciones de los electores de los tres principales candidatos (Fujimori, PPK y Mendoza) siguen el mismo patrón de ubicación que se les asignó a los propios candidatos (ver Gráfico 1), aunque el grado de polarización es menor entre los electores que el que se percibe entre los candidatos.

En las otras dimensiones, las diferencias entre votantes de distintos candidatos son mucho menos marcadas. De los tres candidatos, quien tiene electores menos "estatistas" es PPK, mientras que las diferencias entre Fujimori y Mendoza no son estadísticamente significativas. ${ }^{13}$ Respecto de la escala de discrecionalidad, puede apreciarse que los electores de Keiko Fujimori se ubican en promedio claramente en el lado "discrecional", especialmente si se los compara con los de PPK; en el resto de comparaciones no se encuentran diferencias estadísticamente significativas.

Estos resultados nos indicarían que, entre los electores, las preferencias o actitudes hacia el rol del estado en la economía, o la forma en cómo debe ejercerse

13 No es posible sacar demasiadas conclusiones respecto de los demás candidatos puesto que, teniendo un número menor de electores en la muestra, tienen también un error estándar mayor. 
el poder político no se encuentran claramente alineadas con una dimensión que tiene una carga más ideológico-programática como la diferencia izquierdaderecha. Ello podría reflejar la debilidad o falta de consolidación de vínculos de tipo programático entre ciudadanos y electores, puesto que la combinación de contenidos de hacen la diferencia entre izquierda y derecha entre los políticos, no aparecen claramente en el caso de los votantes.

Gráfico 4. Posición del Elector en las escalas de Izquierda - Derecha, Estatismo y Discrecionalidad, según voto en 1ra vuelta (media e intervalo de confianza al 95\%)
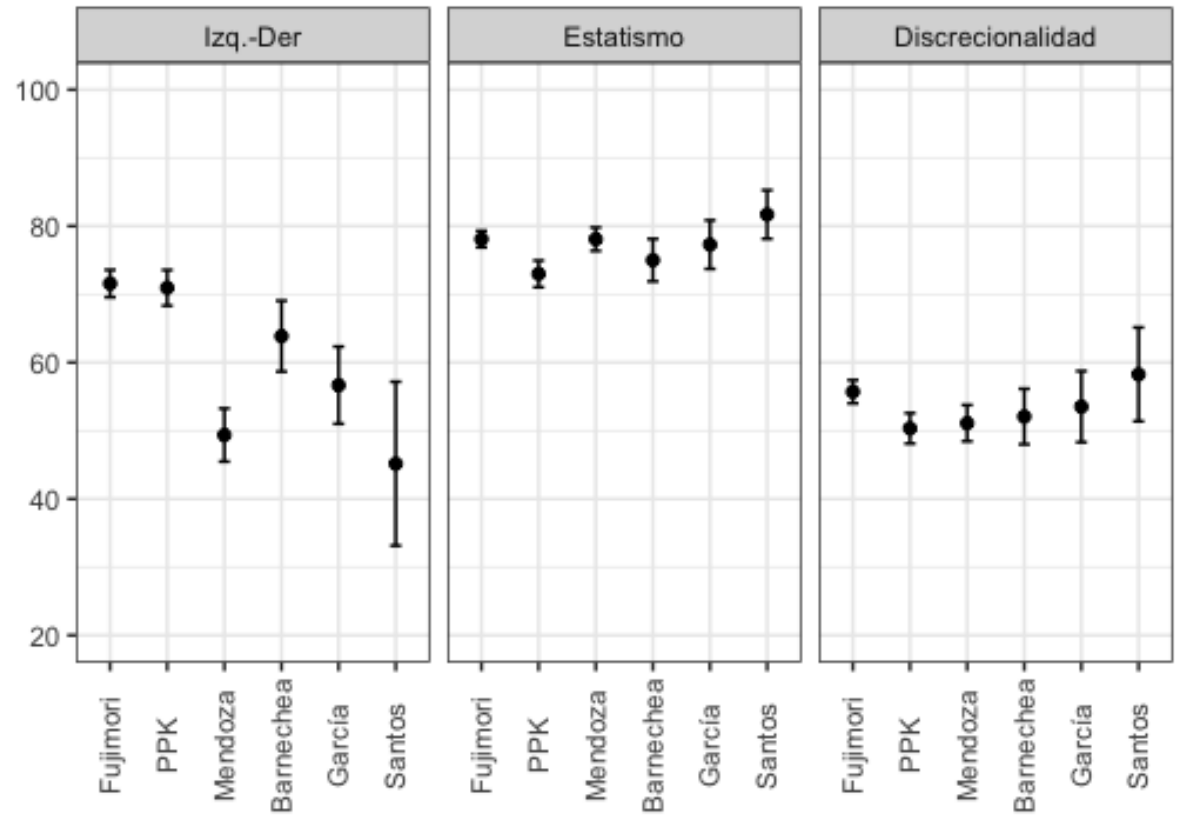

\section{Las evaluaciones programáticas y su efecto electoral}

Un primer indicador de las orientaciones electorales es el nivel de preferencia que se tiene por los diferentes candidatos. En el Gráfico 5 se muestran los niveles de preferencia en una escala de 0 a $100(0=$ no me gusta nada; $100=$ me gusta mucho) de los seis principales candidatos (en las filas) según el voto reportado en primera vuelta (en las columnas). Lo interesante de este gráfico es la jerarquía de preferencias de los votantes de aquellos candidatos que no pasaron a la segunda vuelta. El segundo nivel de preferencias tanto de quienes votaron por Mendoza como por Barnechea era PPK. En el resto de casos no puede determinarse con claridad quien resultaba ser el preferido como "segunda opción", si Fujimori o Kuczynski. De ahí que las últimas semanas de la campaña presidencial resultasen determinantes para inclinar la balanza con un margen estrechísimo a favor de PPK. 
Gráfico 5. Nivel de preferencia por candidatos según voto en 1ra vuelta (media e intervalo de confianza)
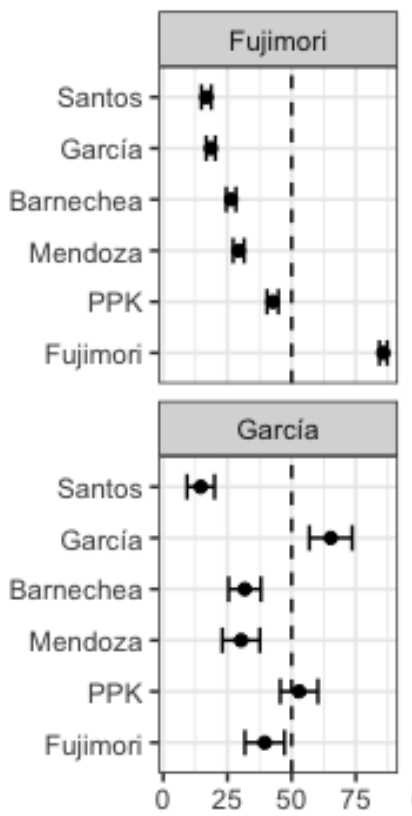
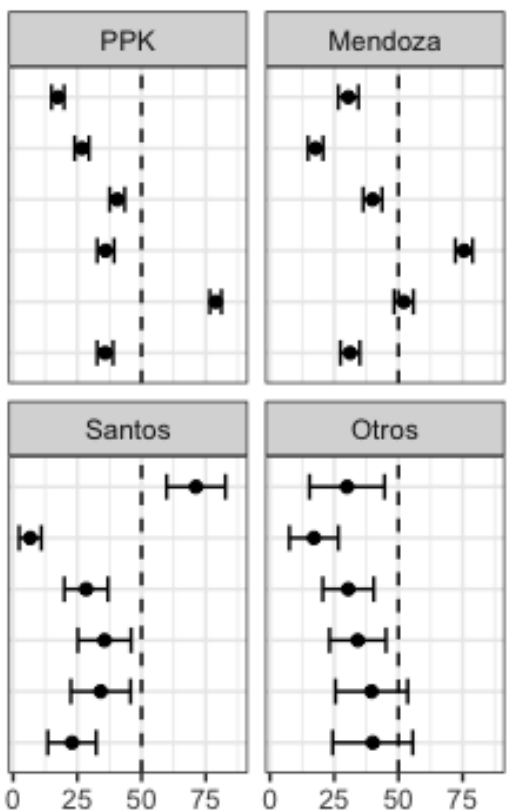
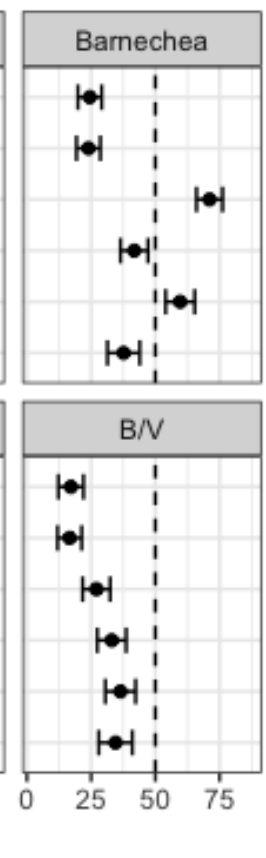

Nivel de preferencia

Con la finalidad de evaluar la relación que tienen las dimensiones que estamos analizando con las preferencias por los tres principales candidatos de la primera vuelta, se realizó un análisis de regresión lineal múltiple (OLS) donde la variable dependiente era el grado de preferencia por cada candidato $(0=$ "no me gusta nada", 10 = "me gusta mucho") y cuyos resultados se muestran en la Tabla 6. Los modelos consideran un conjunto de variables sociodemográficas (sexo, edad, nivel educativo y región de residencia), sin embargo, cuando se incluyen las variables políticas y actitudinales, la mayoría de las variables sociodemográficas tiene un débil poder explicativo. ${ }^{14}$ Como se aprecia en los resultados, la posición ideológica del elector tiene una relación estadísticamente significativa con el nivel de preferencia por los candidatos: cuanto más se ubican los electores hacia la derecha, se incrementa su preferencia por Fujimori y por PPK, lo contrario sucede en el caso de Mendoza.

La escala de estatismo no aparece como relevante para explicar las preferencias por los candidatos, mientras que la de discrecionalidad sí lo es para el caso de Fujimori (cuanto mayor se puntúa en esa escala se incrementan las preferencias por esa candidata). La evaluación que se tiene de los candidatos en los ejes mercado-Estado y ley-discrecionalidad muestran relaciones estadísticamente

14 Se realizó una comparación entre modelos que incluyen y excluyen las variables políticas y actitudinales y se encontró que cuando éstas son tomadas en cuenta los niveles de ajuste (R2) se incrementan de manera bastante significativa. 
significativas con las preferencias por los candidatos. Si el candidato es percibido como proclive a una mayor intervención estatal en la economía, sus niveles de preferencia disminuyen (con excepción de Fujimori, donde no aparece una relación).

El efecto más importante que registran los modelos es la percepción de si el candidato será respetuoso o no de la ley una vez que llegue al poder: si se evalúa que no, sus niveles de preferencia disminuyen de manera significativa. Como se dijo previamente, esto último puede reflejar un efecto endógeno: si un candidato me gusta le atribuyo características que pueden considerarse como positivas, como el "respeto a la ley". Sin embargo, ello también implicaría que el "respeto a la ley" es considerado como una característica deseable, por lo tanto, puede ser tomada en cuenta para decidir qué tanto un candidato es preferible respecto de otro que no es mi primera opción.

Tabla 6. Análisis de regresión para preferencia por candidatos

\begin{tabular}{|c|c|c|c|}
\hline \multicolumn{4}{|c|}{$\begin{array}{l}\text { Modelos OLS para preferencia por principales candidatos } \\
\text { Var. Dependiente: Le gusta (100) - No le gusta (0) el candidato }\end{array}$} \\
\hline & \multicolumn{3}{|c|}{ Candidatos } \\
\hline Var. Independientes & Fujimori & PPK & Mendoza \\
\hline \multirow[t]{2}{*}{ Sexo = Fem } & $3,528^{* *}$ & 0,292 & $-3,693^{* *}$ \\
\hline & $(1,505)$ & $(1,451)$ & $(1,492)$ \\
\hline \multirow[t]{2}{*}{ Edad } & 0,046 & $-0,043$ & $-0,068$ \\
\hline & $(0,049)$ & $(0,047)$ & $(0,048)$ \\
\hline \multirow[t]{2}{*}{ Nivel Educativo } & $-1,113^{* * *}$ & 0,560 & $-0,194$ \\
\hline & $(0,393)$ & $(0,378)$ & $(0,383)$ \\
\hline \multirow[t]{2}{*}{ Región: Norte (a) } & 0,063 & $-6,220^{* * * 4}$ & $-4,514^{* *}$ \\
\hline & $(1,910)$ & $(1,884)$ & $(1,885)$ \\
\hline \multirow[t]{2}{*}{ Región: Centro } & $-1,174$ & $-2,570$ & 4,703 \\
\hline & $(3,099)$ & $(3,010)$ & $(2,984)$ \\
\hline \multirow[t]{2}{*}{ Región: Sur } & $-1,072$ & 1,501 & 1,486 \\
\hline & $(2,243)$ & $(2,146)$ & $(2,274)$ \\
\hline \multirow[t]{2}{*}{ Región: Oriente } & $-3,855$ & 2,025 & $5,193^{*}$ \\
\hline & $(3,022)$ & $(2,949)$ & $(3,098)$ \\
\hline \multirow[t]{2}{*}{ Cand.: Ley - Disc. } & $-0,659^{* * *}$ & $-0,481^{* * *}$ & $-0,515^{* * *}$ \\
\hline & $(0,024)$ & $(0,028)$ & $(0,029)$ \\
\hline \multirow[t]{2}{*}{ Cand.: Mdo. - Estado } & $-0,004$ & $-0,099^{* * * *}$ & $-0,170^{* * * *}$ \\
\hline & $(0,026)$ & $(0,028)$ & $(0,030)$ \\
\hline \multirow[t]{2}{*}{ Discrecionalidad } & $0,118^{* * *}$ & 0,028 & 0,028 \\
\hline & $(0,041)$ & $(0,040)$ & $(0,040)$ \\
\hline
\end{tabular}




\begin{tabular}{lccc}
\hline Estatismo & $-0,021$ & $-0,021$ & 0,010 \\
& $(0,053)$ & $(0,051)$ & $(0,052)$ \\
Izquierda - Derecha & $0,166^{* * *}$ & $0,150^{* * *}$ & $-0,195^{* * *}$ \\
& $(0,030)$ & $(0,029)$ & $(0,030)$ \\
Constante & $72,606^{* * *}$ & $66,618^{* * *}$ & $97,463^{* * *}$ \\
No. Casos Válidos & 1.161 & 1.166 & 1.069 \\
$\mathrm{R}^{2}$ Ajustado & 0,485 & 0,307 & 0,383 \\
\hline
\end{tabular}

Notas: (a) Para las regiones geográficas se toma como referencia la categoría de "Lima - Callao". Errores estándar de los coeficientes entre paréntesis.

${ }^{*} \mathrm{p}<0,05 ;{ }^{* *} \mathrm{p}<0,01 ;{ }^{* * *} \mathrm{p}<0,001$

Las relaciones identificadas en la tabla anterior se trasladan a la decisión electoral en el voto en primera vuelta: ${ }^{15}$ especialmente la ubicación izquierda derecha del elector y la evaluación de los candidatos en el eje ley-discrecionalidad para el conjunto de los candidatos. En el caso específico de Fujimori ello también ocurre con las actitudes favorables a la discrecionalidad.

Finalmente se calcularon tres modelos logísticos binomiales para evaluar la relación entre las actitudes y evaluaciones políticas de los electores y la intención de voto en segunda vuelta. Para ello se excluyeron todos los casos de encuestados que al momento de la encuesta no habían decidido su voto por alguno de los dos candidatos finalistas, por lo que la variable dependiente era si se tenía la intención de votar por Fujimori (1) o por PPK (0). Los dos primeros modelos consideran a todos esos electores, mientras que el tercero sólo toma en cuenta a quienes votaron por otros candidatos diferentes a Fujimori o PPK en la primera vuelta. Como variables independientes el modelo 1 considera las variables políticas que se analizaron en las regresiones de la Tabla 6. En los modelos 2 y 3 se añade la variable que busca medir el anti fujimorismo de los electores. 
Tabla 7. Modelos de regresión logística para voto en segunda vuelta

\begin{tabular}{|c|c|c|c|}
\hline \multicolumn{4}{|c|}{ Modelos Logit Binomial para Voto en Segunda Vuelta } \\
\hline \multicolumn{4}{|c|}{ Var. Dependiente: Vota por Fujimori (1) / Vota por PPK (0) } \\
\hline & \multicolumn{3}{|c|}{ Modelos } \\
\hline Var. Independientes & Modelo 1 & Modelo 2 & $\begin{array}{c}\text { Modelo } 3 \\
\text { Otros 1v. }\end{array}$ \\
\hline \multirow{2}{*}{ Fujimori: Ley - Disc. } & $-0,061^{* * *}$ & $-0,052^{* *}$ & $-0,061^{* * *}$ \\
\hline & $(0,004)$ & $(0,005)$ & $(0,014)$ \\
\hline \multirow[t]{2}{*}{ Fujimori: Mdo. - Estado } & $-0,003$ & $-0,003$ & 0,002 \\
\hline & $(0,004)$ & $(0,004)$ & $(0,010)$ \\
\hline \multirow[t]{2}{*}{ PPK: Ley - Disc. } & $0,043^{* * *}$ & $0,046^{* * *}$ & $0,069^{* * *}$ \\
\hline & $(0,005)$ & $(0,006)$ & $(0,018)$ \\
\hline \multirow[t]{2}{*}{ PPK: Mdo. - Estado } & $0,009^{* *}$ & $0,012^{*}$ & 0,003 \\
\hline & $(0,004)$ & $(0,005)$ & $(0,012)$ \\
\hline \multirow[t]{2}{*}{ Discrecionalidad } & $0,013^{* * *}$ & 0,009 & $-0,003$ \\
\hline & $(0,005)$ & $(0,006)$ & $(0,015)$ \\
\hline \multirow[t]{2}{*}{ Estatismo } & $-0,006$ & $-0,005$ & $-0,010$ \\
\hline & $(0,007)$ & $(0,007)$ & $(0,018)$ \\
\hline \multirow[t]{2}{*}{ Izquierda - Derecha } & $0,009^{* *}$ & 0,001 & $-0,015$ \\
\hline & $(0,004)$ & $(0,005)$ & $(0,013)$ \\
\hline \multirow[t]{2}{*}{ Antifujimorismo } & & $-3,941^{* * *}$ & $-3,990^{* * *}$ \\
\hline & & $(0,460)$ & $(1,205)$ \\
\hline Constante & 0,002 & 0,661 & 1,311 \\
\hline No. Casos Válidos & 911 & 910 & 212 \\
\hline Log Likelihood & -356.198 & -282.871 & -54.029 \\
\hline Akaike Inf. Crit. & 728,40 & 583,74 & 126,059 \\
\hline Nagelkerke Pseudo $R^{2}$ & 0,602 & 0,711 & 0,626 \\
\hline
\end{tabular}

Notas: Se excluyen las respuestas “Blanco o Viciado" y “No sabe”. Errores estándar de los coeficientes entre paréntesis.

${ }^{*} \mathrm{p}<0,05 ;{ }^{* *} \mathrm{p}<0,01 ;{ }^{* *} \mathrm{p}<0,001$

Si nos fijamos en el primer modelo observamos resultados similares a los de la Tabla 6. Sin embargo, cuando se toma en cuenta las actitudes o posiciones antifujimoristas, desaparece la significancia de las variables izquierda-derecha y de la escala de discrecionalidad del elector. En este caso el antifujimorismo se convierte en la variable más relevante del modelo para explicar la intención de voto en segunda vuelta, seguida de la percepción de qué tanto el candidato será respetuoso de la ley. Si solo se consideran los electores votaron por los candidatos que fueron eliminados en la primera vuelta, se llega a conclusiones similares. 
Estos resultados llevarían a afirmar que, en la segunda vuelta electoral, el antifujimorismo como "anti-identidad política" fue un factor central en la derrota de esa candidata. Sin embargo, ese antifujimorismo también tiene algunas bases programáticas: así como una evaluación favorable al fujimorismo está asociada con actitudes discrecionales; lo contrario sucede con el antifujimorismo.

\section{CONCLUSIONES}

Para que un razonamiento programático alimente la decisión electoral, los políticos deben ser capaces de diferenciarse en esos términos y los electores también deben poder darse cuenta de ello. Los resultados de este estudio apuntan a confirmar la presencia de estos procesos en la dinámica política electoral peruana. Cuando los electores logran establecer diferencias significativas entre los candidatos, las asocian con determinadas posiciones que dan contenido a lo que es izquierda y derecha. Las posiciones de izquierda de perciben como favorables a una intervención del Estado en la economía, mientras que las de derecha con una mayor promoción de la libre economía de mercado. Las posiciones de izquierda son vistas como menos apegadas al respeto de normas constitucionales, mientras que lo contrario ocurre en el caso de las de derecha.

Sin embargo, cuando se analiza la manera en cómo los electores se ubican a sí mismos en la escala izquierda-derecha, no se encuentran asociaciones importantes con las escalas de estatismo y de discrecionalidad aplicadas a los encuestados. A diferencia de la manera en como son vistos los candidatos, el estatismo y la discrecionalidad no permiten distinguir entre electores de derecha o de izquierda.

Parece existir una desconexión entre lo que define la distinción entre izquierdaderecha en el caso de la oferta política, y lo que diferencia a la "demanda" (los electores) en esa misma dimensión. El único punto en común sería la simpatía con los candidatos: si el candidato con el que se simpatiza es de derecha, el elector se define en esos términos y viceversa. Esto podría significar que el sistema político peruano, si bien ofrece pistas programáticas, no construye vínculos programáticos lo suficientemente fuertes entre electores y políticos, lo que reforzaría la importancia de los vínculos personalistas o carismáticos en la configuración de identificaciones políticas.

Sin embargo, los vínculos personalistas pueden contribuir a la construcción de conexiones programáticas; ello adquiere especial relevancia para el caso del fujimorismo, un movimiento político que se identifica con el apellido de lo que puede llamarse una dinastía política familiar. El fujimorismo ha sido el movimiento o la corriente política que más ha definido las características del debate y confrontación política en la sociedad peruana desde mediados de los años 90 y ha presentado candidatos en todas las elecciones presidenciales desde 1990 hasta 2016 (de las 7 elecciones, en 5 el candidato presidencial llevaba el apellido Fujimori). Sus seguidores se ubican consistentemente en el lado derecho 
del espectro político y tienen actitudes favorables al ejercicio discrecional del poder, que pueden justificar la necesidad de la "mano dura" para resolver problemas sociales.

Si bien se trata de un movimiento personalista, ha demostrado coherencia discursiva y programática a lo largo el tiempo, además de éxito y arraigo popular. Ello podría haber permitido que ciertas actitudes y preferencias en la población lograran asociarse más claramente con determinadas posiciones políticas enarboladas por este movimiento. El fujimorismo le estaría "enseñando" al electorado qué es ser de "derecha": un tipo de derecha popular, asociada a la "mano dura" en la resolución de los problemas sociales y el mantenimiento del "orden", y que, si bien puede ser favorable al liberalismo económico, no es insensible a ciertas demandas redistributivas de la población. El fujimorismo no es solo un líder o un apellido, sino que también representa ciertas expectativas de cómo se debe gobernar, que encuentran eco en varios sectores de la población.

En la medida que el fujimorismo mantenga su identidad política y sus preferencias programáticas puede seguir siendo el actor central que configure y otorgue significados más coherentes y estables a la competencia política en el Perú. De ahí la importancia del antifujimorismo como criterio para la decisión electoral. Detrás del antifujimorismo también hay ciertas orientaciones programáticas, como el aprecio por una serie de libertades civiles y sociales, así como una apuesta por un Estado de Derecho democrático-liberal. A pesar de la ausencia de liderazgo propio, la fuerza política del antifujimorismo ha contribuido ya dos veces a la derrota electoral de Keiko Fujimori.

Aparentemente, la aparición de un voto o comportamiento electoral programático no requiere necesariamente de un sistema de partidos institucionalizado, con una estructura organizativa densa que se extienda y articule en diferentes sectores del electorado. Un comportamiento electoral programático podría construirse a partir de la consistencia y coherencia discursiva de un liderazgo personalista y carismático que logra "durar" y tener éxito en la competencia electoral. Otros líderes carismáticos en América Latina han tenido éxito político y a la vez generado identificaciones programáticas, dándoles contenido a los significados de derecha e izquierda, por fuera de un sistema de partidos institucionalizado: Chávez en Venezuela, Correa en Ecuador, Morales en Bolivia, Uribe en Colombia, por ejemplo.

Finalmente, estas reflexiones podrían llevarnos a pensar que, contrariamente a ciertas expectativas normativas del funcionamiento de la democracia y del gobierno responsable, los vínculos programáticos no son necesariamente incompatibles o irreconciliables con otras formas que construir relaciones estables entre políticos y ciudadanos mediante mecanismos clientelistas o personalistas. 


\section{REFERENCIAS}

Adams, James, Samuel Merrill y Bernard Grofman. 2005. A Unified Theory of Party Competition: A Cross-National Analysis Integrating Spatial and Behavioral Factors. Cambridge: Cambridge University Press.

Badescu, Gabriel y Pauk E. Sum. 2005. "The Importance of Left-Right Orientations in the New Democracies". Recuperado el 4 de noviembre de 2017 de: http:/ / www.cses.org/plancom/2005Taipei/Badescu\&Sum.pdf

Baker, Andy y Kenneth F. Greene. 2015. "Positional Issue Voting in Latin America". En The Latin American Voter: Pursuing Representation and Accountability in Challenging Contexts, editado por Ryan E. Carlin, Matthew M. Singer, y Elizabeth J. Zechmeister. Ann Arbor, MI: University of Michigan Press, 173-194.

Balassa, Bela, Gerardo Bueno, Pedro Pablo Kuczynski y Mario Henrique Simonsen. 1986. Toward Renewed Economic Growth in Latin America. Mexico DF: El Colegio de México.

Bartolini, Stefano. 2005. "La formation des clivages". Revue Internationale de Politique Comparée 12 (1): 9-34.

Best, Robin E. y Michael D. McDonald. 2010. “The Role of Party Policy Positions in the Operation of Democracy". En Citizens, Context and Choice. How Context Shapes Citizen's Electoral Choices, editado por Russell J. Dalton y Christopher Anderson. Oxford: Oxford University Press, 79-102.

Bornschier, Simon. 2009. "Cleavage Politics in Old and New Democracies". Living Reviews in Democracy. Recuperado el 4 de noviembre de 2017 de: https: / / www.ethz.ch/content/ dam/ethz/special-interest/gess/cis/cis-dam/CIS_DAM_2015/WorkingPapers/Living_Reviews_Democracy/Bornschier.pdf

Carrión, Julio F., Patricia Zárate y Elizabeth J. Zechmeister. 2015. Cultura política de la democracia en Perú y en las Américas, 2014: gobernabilidad democrática a través de 10 años del Barómetro de las Américas. Lima: Instituto de Estudios Peruanos- USAID.

Corral, Margarita y Elizabeth J. Zechmeister. 2010. “The Varying Economic Meaning of 'Left' and 'Right' in Latin America". AmericasBarometer Insigths 38.

Cyr, Jennifer y Carlos Meléndez. 2016. “Una exploración de la identidad (y la antiidentidad) política en nivel subnacional: el fujimorismo y el chavismo en perspectiva comparada". En Partidos políticos y elecciones. Representación política en América Latina, editado por Fernando Tuesta Soldevilla. Lima: Jurado Nacional de Elecciones; PUCP; PNUD, 211-228.

Dalton, Russell J. 2010. "Left-Right Orientations, Context, and Voting Choices". En Citizens, Context and Choice: How Context Shapes Citizen's Electoral Choices, editado por Russell J. Dalton y Christopher Anderson. Oxford: Oxford University Press, 103-125.

Dogan, Matteï. 1996. "Clase, religion, parti: triple déclin dans les clivages électoraux en Europe". Revue Internationale de Politique Comparée 3 (3): 515-540.

Downs, Anthony. 1957. An Economic Theory of Democracy. New York: Harper \& Row.

El País. 2016. "Keiko Fujimori promete mano dura contra el crimen en Perú". Recuperado el 10 de mayo de 2017 de: https://elpais.com/diario/2011/05/20/internacional/1305842409_850215.html

GfK. 2016. "Estudio de opinión pública nacional - urbano rural. Marzo II 2016". Recuperado el 10 de mayo de 2017 de: http:/ /www.gfk.com/fileadmin/user_upload/dyna_content/PE/GfK_OP_Marzo_II_2016_-_Intencion_de_voto_5.pdf

Inglehart, Ronald y Hans Dieter Klingemann. 1976. "Party Identification, Ideological Preference and the Left-Right Dimension Among Western Mass Publics". En Party Identification and Beyond, editado por Ian Budge, Ivor Crewe, y Dennis Farlie. New York: John Wiley \& Sons, 243-274.

Instituto de Opinión Pública - PUCP. 2016. "Estudio Nacional Urbano Rural Preelectoral, Marzo 2016." Recuperado el 10 de mayo de 2017 de: http://iop-data.pucp.edu.pe/ busqueda/encuesta/120? 
Ipsos Opinión y Mercado. 2016a. “Opinión Data. Año 16, No. 199”. Recuperado el 10 de mayo de 2017 de: http://www.ipsos.pe/sites/default/files/opinion_data/Opinion\%20 Data\%20Enero\%202016.pdf

Ipsos Opinión y Mercado. 2016b. “Opinión Data. Año 16, No. 203”. Recuperado el 10 de mayo de 2017 de: http://www.ipsos.pe/sites/default/files/opinion_data/OpinionData030416.pdf

Kitschelt, Herbert. 2000. "Linkages Between Citizens and Politicians in Democratic Polities". Comparative Political Studies 33 (6/7): 845-879.

Kitschelt, Herbert, Kirk Hawkins, Juan Pablo Luna, Guillermo Rosas, y Elizabeth J. Zechmeister. 2010. Latin American Party Systems. Cambridge: Cambridge University Press.

Kitschelt, Herbert y Steven Wilkinson. 2007a. "Citizen-Politician Linkages: An Introduction". En Patrons, Clients, and Policies : Patterns of Democratic Accountability and Political Competition, editado por Herbert Kitschelt y Steven Wilkinson. Cambridge-New York: Cambridge University Press, 1-49.

Kitschelt, Herbert y Steven Wilkinson. 2007b. Patrons, Clients, and Policies: Patterns of Democratic Accountability and Political Competition. Cambridge-New York: Cambridge University Press.

Kroh, Martin. 2009. "The Ease of Ideological Voting: Voter Sophistication and Party System Complexity". En The Comparative Study of Electoral Systems, editado por Hans-Dieter Klingemann. Oxford: Oxford University Press, 220-236.

La Primera. 2016. "Keiko: Mano dura contra delincuentes desde el 28". Recuperado el 10 de mayo de 2017 de: http://www.laprimera.pe/keiko-mano-dura-contra-delincuentes-desde-el-28/.

Lachat, Romain. 2008. "The Impact of Party Polarization on Ideological Voting". Electoral Studies 27 (4): 687-698.

Levitsky, Steven y Maxwell Cameron. 2013. "Democracy Without Parties? Political Parties and Regime Change in Fujimori's Peru." Latin American Politics and Society 45 (3): 1-33.

Lipset, Seymour Martin y Stein Rokkan. 1967a. "Cleavage Structures, Party Systems and Voter Alignments: An Introduction". En Party Systems and Voter Alignments: Crossnational Perspectives, editado por Seymour Martin Lipset y Stein Rokkan. New York: The Free Press, 1-64.

Lipset, Seymour Martin y Stein Rokkan. 1967b. Party Systems and Voter Alignments: Cross-National Perspectives. New York: The Free Press.

Martin, Pierre. 2000. Comprendre les évolutions électorales: la théorie des réalignements revisitée. Paris: Presses de la Fondation Nationale des Sciences Politiques.

McNulty, Stephanie. 2017. "Perú 2016: continuidad y cambio en un año electoral". Revista Ciencia Política 37 (2): 563-587.

Meléndez, Carlos. 2012. "Partidos inesperados: la institucionalización del sistema de partidos en un escenario de post colapso partidario. Perú 2001 - 2011." Recuperado el 4 de noviembre de 2017 de: http://www.fes.org.pe/descargasFES/Partidos\%20inesperados\%20C.\%20Melendez.pdf

Meléndez, Carlos. 2013. “Perú: las elecciones de 2011. Populistas e integrados. Las divisiones políticas en un sistema 'partido'". En Elecciones y política en América Latina: 2009-2011, editado por Manuel Alcántara y María Laura Tagina. México, D.F: Instituto Federal Electoral; Cámara de Diputados; Senado de la República; Miguel Ángel Porrúa, 523562.

Merrill, Samuel y Bernard Grofman. 1999. A Unified Theory of Voting: Directional and Proximity Spatial Models. Cambridge: Cambridge University Press.

Norris, Pippa. 2006. Electoral Engineering: Voting Rules and Political Behavior. Cambridge: Cambridge University Press.

Padua, Jorge e Ingvar Ahman. 1979. "Escalas para la medición de actitudes". En Técnicas de investigación aplicadas a las ciencias sociales, editado por Jorge Padua. México DF: Fondo de Cultura Económica, 154-230. 
Página 12. 2016. "Keiko apuesta por la mano dura". Recuperado el 10 de mayo de 2017 de: https:/ / www.pagina12.com.ar/diario/elmundo/4-297828-2016-04-26.html

Roberts, Kenneth M. 2002. "Social Inequalities Without Class Cleavages in Latin America's Neoliberal Era." Studies in Comparative International Development 36 (4): 3-33.

Sniderman, Paul M. y Matthew S. Levendusky. 2007. "An Institutional Theory of Political Choice". En The Oxford Handbook of Political Behavior, editado por Russell J. Dalton y Hans-Dieter Klingemann. Oxford: Oxford University Press, 437-456.

Sulmont, David. 2015. "Voto ideológico y sistema de partidos en América Latina: el peso de la dimensión izquierda-derecha en el comportamiento electoral en Brasil, Chile, México y Perú". Cuadernos de Investigación del IOP-PUCP (12).

Tanaka, Martín. 2005. Democracia sin partidos: Perú, 2000 - 2005. Lima: Instituto de Estudios Peruanos.

Vergara, Alberto. 2007. Ni amnésicos ni irracionales: las elecciones peruanas de 2006 en perspectiva histórica. Lima: Solar.

Williamson, John y Pedro Pablo Kuczynski. 2003. After the Washington Consensus: Restarting Growth and Reform in Latin America. Washington DC: Institute for International Economics.

Zavaleta, Mauricio. 2014. Coaliciones de independientes: las reglas no escritas de la política electoral. Lima: IEP Instituto de Estudios Peruanos.

Zechmeister, Elizabeth J. 2010. "Left-Right Semantics as a Facilitator of Programmatic Structuration". En Latin American Party Systems, editado por Herbert Kitschelt, Kirk Hawkins, Juan Pablo Luna, Guillermo Rosas y Elizabeth J. Zechmeister. Cambridge: Cambridge University Press, 96-118.

Zechmeister, Elizabeth J. 2015. "Left-Rigth Identifications and the Latin American Voter". En The Latin American Voter: Pursuing Representation and Accountability in Challenging Contexts, editado por Ryan E. Carlin, Matthew M. Singer y Elizabeth J. Zechmeister. Ann Arbor, MI: University of Michigan Press, 195-225.

Recibido: 16.12 .2016

Aceptado: 08.02.2018

David Sulmont: Sociólogo y Doctor en Ciencia Política y Gobierno por la Pontificia Universidad Católica del Perú (PUCP), Magíster en Sociología por l'École des Hautes Etudes en Sciences Sociales de París. Es profesor principal del Departamento de Ciencias Sociales de la PUCP. Ha sido director del Instituto de Opinión Pública, coordinador de la Especialidad de Sociología y de la Maestría en Sociología de dicha casa de estudios. Desde el 2006 es responsable del proyecto del Comparative Study of Electoral Systems (CSES) en el Perú y actualmente forma parte del Planning Committee del Módulo 6 del CSES. Sus temas de especialización son el comportamiento electoral, la opinión pública y los estudios sobre racismo y etnicidad en América Latina. Correo electrónico: sulmont@pucp.pe 